\title{
University teaching in prison in the university penitentiary system of Sassari: practices, research and developments during the pandemic
}

\section{La didattica universitaria in carcere nell'ambito del Polo Universitario Penitenziario di Sassari: pratiche, ricerca e sviluppi ai tempi della pandemia}

\author{
Luisa Pandolfia , Emmanuele Farris ${ }^{\mathrm{b}, 1}$ \\ a Università degli Studi di Sassari, Iupandolfi@uniss.it

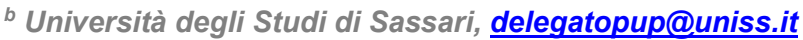

\begin{abstract}
This paper derives from the activities of the University Penitentiary Center of the University of Sassari and aims to explore the impact of the Covid 19 pandemic on university teaching in prison. The subject of penitentiary university teaching is innovative and relevant for educational research and brings into play different skills, professionals, services and institutions. The theoretical framework describes how the right to study in prison is declined on a methodological level. The field research carried out in Sardinia has tried to give the student's voice in prison and the point of view of educators on the educational and organizational impact on university study paths during the pandemic; it is a voice that returns the complexity of a difficult moment, but which also offers useful ideas and stimuli for a more aware restart of the limits and challenges to be faced, as well as good practices to be developed, particularly at the interface between different public institutions as the University and the Penitentiary Administration are.
\end{abstract}

Keywords: university; prison; pandemic; teaching; research.

Sintesi

Il presente contributo nasce nell'ambito dell'attività del Polo Universitario Penitenziario dell'Università di Sassari e si propone di esplorare l'impatto della pandemia da Covid 19 sulla didattica universitaria in carcere. Il tema della didattica universitaria penitenziaria è innovativo e rilevante per la ricerca educativa e chiama in gioco diverse competenze, professionalità, servizi e istituzioni. Il quadro teorico e normativo di riferimento traccia le coordinate in cui si inserisce il diritto allo studio in carcere e ne declina i significati e le implicazioni sul piano metodologico. La ricerca sul campo realizzata in Sardegna ha cercato di dar voce agli studenti detenuti e agli educatori in merito alle ricadute a livello educativo ed organizzativo sui percorsi di studio durante la pandemia; una voce che restituisce la complessità di un momento difficile, ma che offre anche spunti e stimoli utili per una ripartenza più consapevole dei limiti e delle sfide da affrontare, così come delle buone prassi da sviluppare soprattutto all'interfaccia tra istituzioni pubbliche, quali sono l’Università e l'Amministrazione Penitenziaria.

Parole chiave: università; carcere; pandemia; didattica; ricerca.

\footnotetext{
${ }^{1}$ L'attribuzione dei paragrafi è così suddivisa: 1 e 2 a Luisa Pandolfi; 3 a Emmanuele Farris; 4 e 5 a cura di entrambi gli autori.
} 


\section{Introduzione}

La pandemia Covid 19 ha reso necessaria una rimodulazione degli interventi e delle pratiche in differenti contesti educativi e sociali, amplificando, talvolta, nodi critici già esistenti, ma in altri casi innovando ed implementando strategie efficaci. Il sistema della giustizia e, in particolare, quello carcerario, è stato investito in modo complesso ed articolato dall'emergenza sanitaria, con ricadute difficili da gestire. Il segmento approfondito in questa sede riguarda, nello specifico, la formazione universitaria in carcere. Il tema della didattica universitaria in ambito penitenziario è piuttosto recente nel panorama scientifico nazionale e costituisce un terreno di sfida interessante che chiama in gioco la dimensione del lifelong learning e la sperimentazione di nuove metodologie didattiche. Partendo dall'assunto della funzione rieducativa della pena, quale fondamento giuridico stabilito a livello costituzionale su cui si è sviluppato l'ordinamento penitenziario e l'esecuzione penale, si evidenzia come il diretto coinvolgimento delle università nello sviluppo culturale della popolazione detenuta si configuri come impegno didattico, ma anche come 'terza missione', in termini di promozione del dialogo e del benessere della società e di creazione di sinergie e alleanze a livello territoriale ed, infine, anche come impegno nella ricerca scientifica. E proprio quest'ultimo aspetto è la cornice in cui si inserisce il presente contributo, che, partendo dall'esperienza del Polo Universitario Penitenziario dell'Università di Sassari, descrive un'indagine sul campo che ha coinvolto diversi Istituti Penitenziari della regione Sardegna con la finalità di far emergere il punto di vista degli studenti detenuti e dei funzionari giuridico-pedagogici in merito alle ricadute e all'impatto della pandemia Covid 19 sulla didattica universitaria, focalizzando l'attenzione sulle problematiche emerse, sui punti di forza e sugli sviluppi possibili, anche in termini di buone pratiche da valorizzare.

\section{La formazione universitaria in carcere: modelli e finalità rieducative}

L'istruzione è uno degli elementi del trattamento penitenziario e, nello specifico, il D. Lgs. n. 123/2018 Riforma dell'ordinamento penitenziario stabilisce che devono essere agevolati la frequenza e il compimento degli studi universitari e tecnici superiori, anche attraverso convenzioni e protocolli d'intesa con istituzioni universitarie e con istituti di formazione tecnica superiore, nonché l'ammissione dei detenuti ai tirocini. In tal senso, appare opportuno citare le Linee guida del MIUR, adottate con Decreto Interministeriale del 12 marzo 2015, in cui sono previste delle disposizioni in materia di percorsi di istruzione negli istituti di prevenzione e pena, finalizzati a "rieducare il detenuto alla convivenza civile attraverso azioni positive che lo aiutino nella ridefinizione del proprio progetto di vita e nell'assunzione di responsabilità verso se stesso e la società, tenuto conto che l'istruzione costituisce il presupposto per la promozione della crescita culturale e civile del detenuto e la base necessaria alla sua formazione professionale, tecnica e culturale" (MIUR, $2015 \mathrm{p}$. 18). Il documento specifica, altresì, che la programmazione di tali percorsi dovrà tener conto della specificità e distintività dell'istruzione nel sistema penitenziario con l'obiettivo di rendere compatibili gli assetti organizzativi e didattici con i tempi e i luoghi della detenzione e con la specificità degli studenti detenuti, in coerenza con il principio dell'individualizzazione del trattamento penitenziario. In tale prospettiva si colloca anche il protocollo d'intesa tra il MIUR e il Ministero della Giustizia del 2016 denominato Programma speciale per l'istruzione e la formazione negli istituti penitenziari e nei Servizi Minorili della Giustizia in cui si ribadisce l'importanza di promuovere ed attivare la sperimentazione di percorsi certificabili, modulari e flessibili in contenuti e durata, con 
possibilità di prosecuzione anche dopo l'uscita dal circuito detentivo e finalizzati sia a favorire l'acquisizione ed il recupero di abilità e competenze individuali, sia a sviluppare una politica dell'istruzione integrata con la formazione professionale e supportata dalla collaborazione con le Regioni ed il mondo delle imprese. Concentrando l'attenzione sulla formazione universitaria, la letteratura socio-pedagogica sul tema (Brancucci, 2016; Caldin 2018; Decembrotto 2018; Downes, 2014) mette in luce come non possa essere intesa solo nei termini di semplice erogazione dell'offerta formativa e di acquisizione di conoscenze disciplinari, bensì all'interno di una visione più ampia in cui assume rilevanza centrale la promozione personale, sociale e culturale delle persone, i bisogni formativi e il loro patrimonio esperienziale. La formazione universitaria in carcere dovrebbe mirare allo sviluppo di competenze necessarie per la realizzazione personale, l'inclusione sociale, la futura occupabilità e la cittadinanza. L'istruzione ed il successo formativo si configurano, quindi, come importanti fattori protettivi per la ricostruzione di percorsi di vita resilienti e per la fuoriuscita dal circuito della devianza perché offrono la possibilità di ampliare il proprio campo di esperienze, di sperimentare, scoprire e acquisire consapevolezza delle proprie capacità, potenzialità e talenti, confrontandosi con le dimensioni dell'impegno e della responsabilità, potenziando autostima ed autoefficacia (Pandolfi, 2020). Il valore dell'istruzione e della formazione risiede nella loro capacità di investire di nuovi significati ed opportunità il tempo presente, aprendo alla prospettiva temporale del futuro: "Il tempo del carcere non può essere un tempo vuoto o un tempo di attesa della fine della pena, ma luogo dove il tempo assume dei significati attraverso le cose che si fanno: è questo il valore dell'istruzione. Le iniziative scolastiche, infatti, cercano di dare un senso alla detenzione aiutando il ristretto non solo ad acquisire un titolo di studio e apprendere dei contenuti, ma anche ad aprirsi alla riflessività" (Cesaro, 2018, p. 32). In tale ottica, nella didattica universitaria penitenziaria assumono un'importanza centrale quelle che possono essere definite le caratteristiche peculiari della programmazione didattica, ossia: la flessibilità in itinere rispetto a contenuti, interventi, metodi; la concretezza, relativa alla definizione degli obiettivi, alla pianificazione delle attività, all'analisi di fattibilità e alla valutazione dei risultati; l'apertura a nuove possibilità, esperienze, apprendimenti, in una prospettiva di cambiamento, crescita e miglioramento; la partecipazione, intesa come coinvolgimento attivo del soggetto nel percorso educativo e/o di apprendimento e la motivazione, ossia la capacità di costruire condizioni in grado di stimolare e rinforzare l'adesione e la partecipazione del soggetto alle proposte educative e didattiche. Diventa, altresì, necessario collocare la formazione universitaria in carcere nella prospettiva dell'apprendimento permanente, in quanto gli studenti universitari sono spesso adulti in formazione, che riprendono gli studi dopo diversi anni e che sovente hanno avuto l'opportunità di (ri) percorrere le diverse tappe dell'istruzione (dalla scuola secondaria inferiore di primo e secondo grado fino all'Università) proprio all'interno del carcere. A tal proposito, Knowles (1993) descrive alcuni principi dell'apprendimento adulto che possono rappresentare delle utili chiavi di lettura per orientare la didattica universitaria in ambito penitenziario:

- la graduale autonomia del soggetto in apprendimento;

- l'importanza dell'esperienza e della propria traiettoria biografica, che rende gli adulti in apprendimento essi stessi risorsa nel percorso formativo;

- un orientamento centrato sul problema, anziché solo sui contenuti disciplinari;

- un bisogno di conoscere e di sapere a che cosa può servire quella specifica conoscenza e come può essere applicata a livello operativo;

- una motivazione ad apprendere che spesso è legata all'autostima, al raggiungimento di obiettivi nel proprio percorso di vita, al miglioramento di sé. 


\section{II Polo Universitario Penitenziario dell'Università di Sassari nel contesto italiano}

Il bisogno e la domanda di percorsi di alta formazione provenienti da persone detenute negli Istituti Penitenziari (II.PP.) italiani è certamente antecedente rispetto alla costituzione dei Poli Universitari Penitenziari (PUP), che ha luogo, mediante la sottoscrizione di opportuni protocolli d'intesa tra Università e Amministrazione Penitenziaria (Direzioni degli II.PP., Provveditorati dell'Amministrazione penitenziaria (PRAP) o entrambi), a partire dalla fine degli anni '90 con la costituzione del primo PUP presso l'Università di Torino, che sancisce la formalizzazione di rapporti continuativi e la sottoscrizione di regole scritte per la presenza del personale universitario (docente, ma anche amministrativo e talvolta tecnico e bibliotecario) all'interno degli istituti penitenziari (Prina, 2018; 2020a). Successivamente, anche sotto la spinta della normativa, a partire dagli anni 2000 vengono costituiti formalmente numerosi Poli Universitari Penitenziari, che dall'Aprile 2018 si costituiscono in una Conferenza Nazionale dei Poli Universitari Penitenziari Italiani (CNUPP) in seno alla Conferenza dei Rettori delle Università Italiane (CRUI), che comprende 30 atenei operativi in circa 75 istituti penitenziari e coinvolge circa 800 persone detenute (Prina, 2020b). Sebbene l'inizio delle attività didattiche negli istituti penitenziari da parte dei docenti dell'ateneo sassarese risalga almeno agli anni Novanta, il Polo Universitario Penitenziario dell'Università di Sassari (PUP Uniss) nasce tra l'ateneo e il Provveditorato Regionale dell'Amministrazione Penitenziaria, sotto l'egida del Dipartimento dell'Amministrazione Penitenziaria del Ministero della Giustizia (DAP) e del Dipartimento della Giustizia Minorile e di Comunità del Ministero della Giustizia (DGMC), tutti enti con i quali l'Università di Sassari ha stipulato protocolli d'intesa a partire dagli anni 2000, volti alla definizione dei rispettivi ruoli per facilitare lo studio universitario alle persone ristrette negli istituti penitenziari. Il Polo Universitario Penitenziario viene formalmente costituito con la firma del protocollo d'intesa tra Uniss e PRAP del 26 Marzo 2014, che individua quali sedi penitenziarie di attività didattica universitaria la Casa di Reclusione di Alghero, la Casa Circondariale di Nuoro, la Casa Circondariale di Sassari e la Casa di Reclusione di Tempio-Pausania (Farris \& Guido, 2020). Il Protocollo, scaduto il 26 Marzo 2020, è stato rinnovato a Settembre-Ottobre 2020 e vede la partecipazione, oltre che di Uniss e PRAP, anche dell'Ufficio Interdistrettuale di Esecuzione Penale Esterna di Cagliari (UIEPE) e del Centro per la Giustizia Minorile (CGM) per la Sardegna. Da maggio 2017 il PUP Uniss si è dotato di un nuovo regolamento all'avanguardia in campo nazionale, in cui le tutele previste sono riconosciute non solo ai carcerati ma anche a tutte le persone con qualunque limitazione alla libertà personale (arresti domiciliari, libertà vigilata) e per tutta la durata del corso di studi, anche qualora fosse conclusa la pena. Dall'estate 2017 è stata allestita e resa fruibile una pagina web dedicata (https://www.uniss.it/polo-penitenziario) che viene implementata continuamente. Per meglio radicare la presenza nei quattro Istituti Penitenziari e sul territorio regionale in cui opera, il PUP Uniss dal 2017-18 è la prima realtà italiana ad avere istituzionalizzato attività di public engagement in carcere, con un ciclo seminariale annuale tematico, concepito con i detenuti e la direzione penitenziaria di Alghero sul tema della "Ruralità: criticità e opportunità" nel 2017-18 e sul "Comparto Ittico" nel 2018-19. Con le donne detenute nella Casa Circondariale di Sassari nel 2019 è invece stato realizzato un percorso di conoscenza personale e recupero dell'autostima intitolato "Ricomincio da me". L'accesso ai materiali di studio per gli studenti in regime di detenzione viene sempre migliorato grazie alle sinergie con il Sistema Bibliotecario di Ateneo e l'Ente Regionale per il diritto allo Studio Universitario (ERSU) di Sassari, che da oltre dieci anni concede all'Università un importante contributo annuale per l'acquisto dei testi di studio necessari 
agli studenti detenuti. Sono attivi servizi di orientamento, supporto amministrativo per le immatricolazioni, accoglienza in ingresso, costantemente implementati e aggiornati (https://www.uniss.it/uniss-comunica/polo-penitenziario/servizi). L'ateneo sassarese, che ha adottato dall'anno accademico 2018-19 un Regolamento Carriere Studenti molto inclusivo, con servizi e tutele dedicate agli studenti con esigenze speciali (SES) come gli studenti in regime di detenzione (ma anche disabili, studenti infortunati, studentesse in stato di gravidanza, persone vittime di reato, persecuzioni, calamità e altro), ha visto premiati $i$ suoi sforzi con l'erogazione da parte del Ministero dell'Istruzione, dell'Università e della Ricerca, di un finanziamento speciale di $€ 220.000$ appositamente destinato all'implementazione delle attività del Polo Universitario Penitenziario, nell'ambito del Fondo di Finanziamento Ordinario delle Università 2017, D.M. n. 610/2017 art. 10 "Ulteriori interventi" per il progetto Implementazione delle attività del Polo Universitario Penitenziario. Successivamente, la Fondazione di Sardegna il 31 Gennaio 2020 ha concesso al PUP Uniss un contributo di $€ 12,000$ quale intervento per il Progetto Potenziamento dei servizi didattici per persone detenute iscritte all'Università di Sassari. Nell'anno accademico 2019-20 il PUP dell'Università di Sassari è risultata la quarta realtà italiana per numero di iscritti (66), con un incremento del $+61 \%$ rispetto al dato medio riferito al triennio precedente, e prima per incidenza sulla popolazione detenuta locale (5.4\% contro una media nazionale dell' $1.4 \%$ ) (Comitato Didattico del PUP Uniss, 2020). I 66 studenti sono così ripartiti:

- iscritti al I anno: 35 studenti (di cui 4 inattivi);

- iscritti al II anno: 16 studenti (di cui 1 inattivo);

- iscritti al III anno: 5 studenti (tutti attivi);

- iscritti Fuori corso: 8 studenti (di cui 2 inattivi);

- Laureandi: 2 studenti (entrambi laureati nel 2020).

Il $61 \%$ degli studenti afferisce al circuito di Alta Sicurezza, 1'8.5\% al 41bis e "solo" il $30.5 \%$ al circuito di Media Sicurezza.

La ripartizione nei diversi Istituti Penitenziari è riportata nella successiva Figura 1.

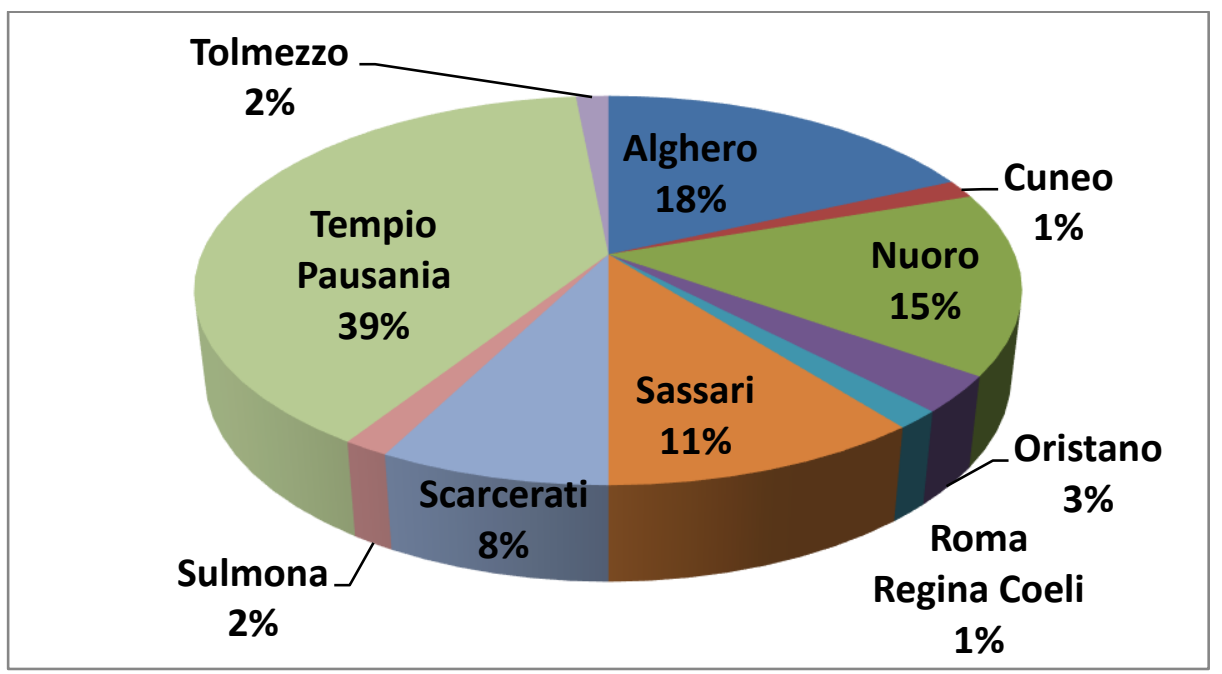

Figura 1. Percentuale di studenti in regime di detenzione nei diversi II.PP. e in esecuzione penale esterna iscritti nell'anno accademico 2019-20 all'Università di Sassari. 
L'incidenza di studenti universitari rispetto alla popolazione detenuta al 29.02.2020 (fonte DAP, Min. Giustizia), è del 17.3\% nella Casa di reclusione di Tempio Pausania, 8.5\% nella Casa di Reclusione di Alghero, 3.1\% nella Casa Circondariale di Nuoro e dell'1.9\% nella Casa Circondariale di Sassari.

Gli studenti in regime di detenzione iscritti all'Università di Sassari nel 2019-20 studiano in 15 corsi di laurea differenti, ripartiti in 5 dipartimenti (Figura 2).

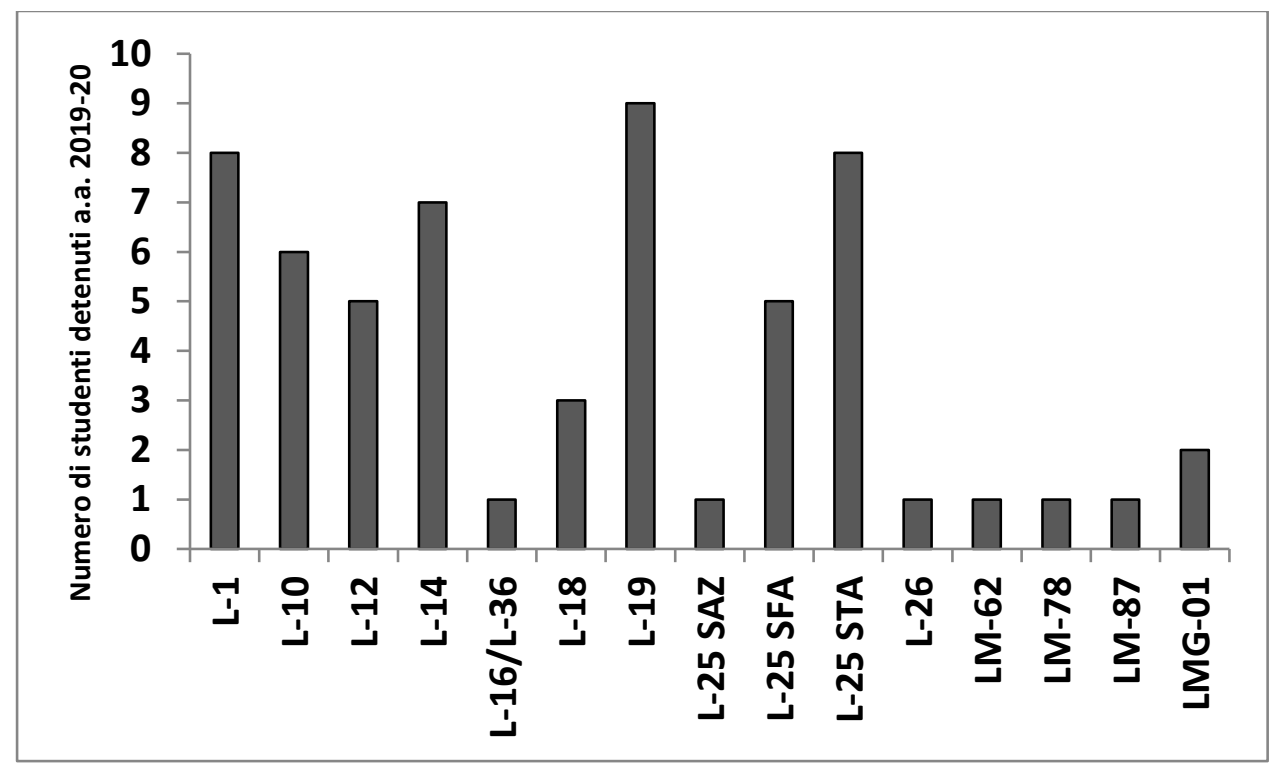

Figura 2. Distribuzione per Corso di Laurea dei 59 studenti detenuti che hanno completato l'anno accademico 2019-20.

Per ciò che concerne le performance degli studenti afferenti al Polo Universitario Penitenziario dell'Università di Sassari nell'anno accademico 2019-20, la media dei CFU acquisiti dagli studenti in regime di detenzione nell'anno accademico 2019-20 (monitoraggio effettuato nel periodo 10 agosto 2019-10 Agosto 2020) è stata di 21.9 CFU/studente, con un incremento del $+12.5 \%$ rispetto al triennio precedente. Il $7.8 \%$ degli studenti non ha sostenuto esami nell'anno accademico 2019-20 (quindi non ha conseguito CFU nel periodo 10 agosto 2019-10 agosto 2020); il 17.6\% ha conseguito tra uno e nove CFU; il $41.2 \%$ ha conseguito tra 10 e 25 CFU e il $33.3 \%$ ha conseguito più di 25 CFU. Il numero medio più alto di CFU conseguiti nel periodo di osservazione è stato di $43.5 \mathrm{CFU}$ da parte degli studenti del III anno; seguono gli studenti laureandi con 28.5 CFU/anno; gli studenti del II anno e fuori corso con 22.1 e 22.2 CFU medi annui, rispettivamente e infine gli studenti del I anno con 17.9 CFU/anno. Sono state riscontrate anche notevoli differenze relative al luogo di detenzione: gli studenti in esecuzione penale esterna hanno acquisito in media 45 CFU/anno; i detenuti in II.PP. extraregionali 28.7 CFU/anno; i detenuti nella CR Tempio Pausania 26.8 CFU/anno, i detenuti nella CR Alghero 14.9 CFU/anno, e infine i detenuti nelle case circondariali di Nuoro e Sassari 13.8 e 13.5 CFU/anno rispettivamente. Sembra incidere anche il circuito detentivo: le performances medie migliori sono quelle dei detenuti in AS che hanno acquisito 25.3 CFU/anno, seguiti dai detenuti in regime ex art. 41 bis O.P. con $21.3 \mathrm{CFU} /$ anno e infine i detenuti in media sicurezza hanno riportato in media 15.6 CFU/anno. Infine, anche il dipartimento universitario di afferenza sembra un fattore significativo nel determinare la performance annuale degli studenti detenuti: gli studenti iscritti al Dipartimento di Storia, Scienze dell'Uomo e della Formazione (DISSUF) 
hanno acquisito in media 28.7 CFU/anno; gli studenti afferenti ai corsi del Dipartimento di Scienze Economiche e Aziendali (DISEA) 25.5 CFU/anno; seguono gli studenti iscritti ai corsi dei dipartimenti di Giurisprudenza e Scienze Umanistiche e Sociali con 19.7 e 19.5 CFU/anno rispettivamente, e infine gli studenti dei corsi del Dipartimento di Agraria con $11 \mathrm{CFU} / \mathrm{anno.}$

\section{Un'indagine sul campo sull'andamento della didattica universitaria penitenziaria in Sardegna durante la pandemia}

L'indagine, realizzata nel periodo luglio-settembre 2020, è stata promossa all'interno del Polo Universitario Penitenziario dell'Università di Sassari, grazie alla collaborazione del PRAP e delle Direzioni e Aree Educative-Trattamentali dei quattro Istituti Penitenziari sardi che afferiscono al PUP Uniss, e ha preso le mosse dalla seguente domanda di ricerca: come è stata affrontata la sfida della didattica a distanza durante la pandemia in ambito penitenziario e quali le ricadute a livello educativo ed organizzativo? Nei successivi punti in cui si articola il paragrafo verranno descritti il contesto di riferimento della ricerca e i vari aspetti metodologici in cui si declina.

\subsection{Il contesto}

Con comunicazioni scritte inviate all'Università il 5 marzo 2020, le Direzioni penitenziarie di Alghero e Nuoro sospendevano l'attività didattica in presenza del personale Uniss nei due istituti penitenziari. I contemporanei e successivi provvedimenti del Governo (DPCM dell'8 marzo 2020) decretavano la sospensione degli accessi di personale esterno a tutti gli II.PP. del territorio nazionale. Il Delegato rettorale per il PUP Uniss produceva un comunicato inserito nella pagina web del PUP (https://www.uniss.it/polo-penitenziario) in cui sono inserite una lettera per gli studenti detenuti e un comunicato del Rettore. Il Rettore dell'Università di Sassari, con nota prot. n. 31001/2020 indirizzata al PRAP Sardegna e alle Direzioni degli II.PP. afferenti al protocollo, chiedeva che venissero comunque garantiti i seguenti servizi a beneficio dei detenuti studenti iscritti all'Università di Sassari:

- consegna agli studenti dei testi e altro materiale di studio, in formato cartaceo o digitale su cd non riscrivibili, tramite i Funzionari giuridico pedagogici o altro personale dell'Amministrazione Penitenziaria;

- possibilità di effettuazione di esami di profitto ed eventualmente di laurea, tramite collegamento remoto mediante sistema di videoconferenza Lynch o via Skype;

- possibilità di colloqui tra detenuti e responsabili della didattica universitaria (personale docente, amministrativo, tutor), anche attraverso la mediazione del personale degli Istituti penitenziari, tramite collegamenti telematici come per gli esami.

Il PRAP Sardegna, con nota prot. n. 05824/2020 rispose al Rettore Uniss che, permanendo la sospensione dell'accesso agli II.PP. di tutto il personale Uniss incluso quello non strutturato (tutor), non riteneva percorribile l'ipotesi di far sostenere colloqui o esami tramite videoconferenza con Skype, poiché ogni risorsa in tal senso sarebbe dovuta essere destinata all'effettuazione dei colloqui con i familiari. Contemporaneamente il 12 marzo 2020 il Presidente CNUPP, su sollecito del Delegato rettorale per il PUP Uniss, scriveva al Direttore generale dei detenuti e del trattamento dell'Amm.ne Penitenziaria, al Capo Dipartimento e al Direttore Ufficio II - Trattamento e Lavoro penitenziario, ottenendo risposta positiva dalla Direzione Generale dei Detenuti e del Trattamento con Circolare 
GDAP n. 0084702.U del 12.03.2020 indirizzata ai Provveditori regionali, cui seguiva la Nota del PRAP Cagliari N. 008211/U.D. e T./S8 del 05.04.2020. La Circolare GDAP n.0084702.U del 12.03.2020 invitava i Provveditori regionali a comunicare alle Direzioni degli II.PP. che possono consentire lo svolgimento degli esami di laurea, esami universitari e colloqui didattici tra docenti e studenti detenuti, sia appartenenti ai circuiti di Media Sicurezza che AS3, mediante videoconferenza e/o tramite Skype. La circolare, allo scopo di limitare il disagio dei detenuti sopra indicati, in particolare se iscritti a corsi universitari, consentiva anche l'uso della posta elettronica per comunicazioni celeri con i docenti (previsione peraltro non applicata e non strettamente necessaria in Sardegna, dato il costante rapporto tra staff del PUP e staff FGP degli II.PP.). Conseguentemente a queste disposizioni del DAP e del PRAP i quattro II.PP. afferenti al PUP Uniss si sono attivati per stabilire, con intensità e frequenza diversificate, modalità di colloqui ed esami per gli studenti ivi detenuti: la CC Nuoro con nota scritta Prot. 5981/6/SAC del 13 maggio 2020 indicando la piattaforma Teams; la CR Tempio con comunicazione scritta Prot. 2537 del 20 maggio 2020 indicando la piattaforma Teams. La CR Alghero ha invece dato comunicazioni per le vie brevi, realizzando il primo esame orale a distanza il 30 aprile 2020 (Filosofia del Linguaggio per Mediazione Linguistica e Culturale) e attivando colloqui regolari con docenti e tutor a partire dal lunedì 11 maggio 2020 su piattaforma Skype for Business. Infine, la CC Sassari contemporaneamente attivava, per le vie brevi, possibilità di colloqui via skype nella seconda decade di maggio 2020. L'attività di didattica a distanza si è svolta in maniera più regolare presso la CR Alghero, dove, tra l'11 maggio $2020 \mathrm{e}$ settembre 2020, si sono svolti ben 66 colloqui (inclusi esami orali). L'enorme sforzo sviluppato dal personale Uniss insieme a quello dell'Amministrazione Penitenziaria, ha permesso non solo di garantire il regolare svolgimento degli esami di profitto (dapprima solo in forma scritta, tra il 5 marzo e il 10 maggio 2020, poi anche in forma orale a distanza) e di un esame di laurea in svolto a distanza tra la commissione e la sede penitenziaria di Tempio Pausania a luglio 2020, ma anche di far acquisire più CFU rispetto ai mesi precedenti (Figura 3). Anche se questo dato è ovviamente influenzato dagli studenti del I anno, che non potevano sostenere esami tra il 10 agosto 2019 e il gennaio 2020, tuttavia è significativo che i 51 studenti detenuti, regolarmente iscritti a Uniss, che hanno completato l'anno accademico, abbiano acquisito il $61.5 \%$ dei CFU annui in un intervallo di tempo inferiore alla metà dell'anno, ovvero nel periodo interessato dall'emergenza Covid (1.03.2020-10.08.2020).

\begin{tabular}{|l|c|c|c|}
\hline & CFU 12 mesi $^{2}$ & CFU Covid-19 & $\begin{array}{c}\text { \% CFU Covid / } \\
\text { Anno }\end{array}$ \\
\hline I anno $=26$ & 466 & 360 & 77.2 \\
\hline II anno $=13$ & 288 & 158 & 54.9 \\
\hline III anno $=4$ & 174 & 120 & 69.0 \\
\hline Fuori corso $=6$ & 133 & 35 & 26.3 \\
\hline Laureandi $=2$ & 57 & 15 & 26.3 \\
\hline Totale $=51$ & 1118 & 688 & 61.5 \\
\hline
\end{tabular}

Figura 3. CFU acquisiti su base annua e successivamente al 1.03.2020 dagli studenti del PUP Uniss: dati assoluti per anno di corso e percentuale CFU Covid sul totale.

\footnotetext{
${ }^{2}$ Dal 10.08.2019 al 10.08.2020

${ }^{3}$ Dal 01.03 .2020 al 10.08 .2020
} 
Anche sulle performance del periodo interessato alla pandemia incide l'istituto di residenza (Figura 4): rispetto alla media complessiva $(61.5 \%)$ risultano avere una performance migliore CC Nuoro (82.6\%) e CR Tempio Pausania (66.3); risultano in linea con la media altri II.PP (62.6\%) e CR Alghero (58.5\%) e sono sotto media CC Sassari (34.6\%) e gli studenti in esecuzione penale esterna $(33.3 \%)$.

\begin{tabular}{|l|c|c|c|}
\hline & ${\text { CFU 12 } \text { mesi }^{4}}^{4}$ & CFU Covid-19 & $\begin{array}{c}\text { \% CFU Covid / } \\
\text { Anno }\end{array}$ \\
\hline Alghero CR $=10$ & 164 & 96 & 58.5 \\
\hline Nuoro CC $=5$ & 69 & 57 & 82.6 \\
\hline Sassari CC $=6$ & 81 & 28 & 34.6 \\
\hline Tempio Pausania CR =24 & 644 & 427 & 66.3 \\
\hline Altri II.PP. $=3$ & 91 & 57 & 62.6 \\
\hline Esecuz. Penale Esterna $=3$ & 69 & 23 & 33.3 \\
\hline Totale $=51$ & 1118 & 688 & 61.5 \\
\hline
\end{tabular}

Figura 4. CFU acquisiti su base annua e successivamente al 1.03.2020 dagli studenti del PUP Uniss: dati assoluti per II.PP. e percentuale CFU Covid sul totale.

Un altro fattore molto importante che ha inciso sulle performance del periodo interessato dalla pandemia è stato il regime di detenzione (Figura 5): rispetto alla media complessiva $(61.5 \%)$ risultano avere una performance migliore il regime 41 bis $(74,1 \%)$ e il circuito AS (67.9); risultano sotto media gli studenti in media sicurezza (47\%) e quelli in esecuzione penale esterna (33.3\%). Queste percentuali hanno portato alla fine del periodo annuale considerato (agosto 2019 - agosto 2020) gli studenti detenuti in AS ad avere la performance media più alta (24.6 CFU/studente) seguiti da quelli in esecuzione penale esterna (23 $\mathrm{CFU} /$ studente), i 41 bis (21.2 CFU/studente) e per ultimi quelli in MS (16.7 CFU/studente). Se confrontiamo questo dato con quello relativo all'anno accademico 2018-19 (Comitato Didattico del PUP Uniss, 2019) possiamo notare come prima della pandemia la media dei CFU per studente sia più alta (anche se di poco) in media sicurezza che in alta sicurezza $(\mathrm{MS}=17.5 \mathrm{CFU} / \mathrm{studente} ; \mathrm{AS}=16.3 \mathrm{CFU} /$ studente; $41 \mathrm{bis}=12.1 \mathrm{CFU} /$ studente; Esecuzione Penale Esterna $=4.7 \mathrm{CFU} /$ studente).

\begin{tabular}{|l|c|c|c|}
\hline & ${\text { CFU 12 } \text { mesi }^{6}}$ & CFU Covid-19 $^{7}$ & $\begin{array}{c}\text { \% CFU Covid / } \\
\text { Anno }\end{array}$ \\
\hline 41bis $=4$ & 85 & 63 & 74.1 \\
\hline $\mathrm{AS}=29$ & 713 & 484 & 67.9 \\
\hline $\mathrm{MS}=15$ & 251 & 118 & 47.0 \\
\hline Esecuz. Penale Esterna $=3$ & 69 & 23 & 33.3 \\
\hline Totale $=51$ & 1118 & 688 & 61.5 \\
\hline
\end{tabular}

Figura 5. CFU acquisiti su base annua e successivamente al 1.03.2020 dagli studenti del PUP Uniss: dati assoluti per circuito detentivo e percentuale CFU Covid sul totale.

Infine, un ultimo fattore decisivo sulle performance del periodo interessato alla pandemia è stato il dipartimento universitario di afferenza (Figura 6): rispetto alla media complessiva

\footnotetext{
${ }^{4}$ Dal 10.08.2019 al 10.08.2020

${ }^{5}$ Dal 01.03.2020 al 10.08.2020

${ }^{6}$ Dal 10.08.2019 al 10.08.2020

${ }^{7}$ Dal 01.03.2020 al 10.08.2020
} 
$(61.5 \%)$ risultano avere una performance migliore gli studenti afferenti al Dipartimento di Scienze Economiche e Aziendali (DISEA) (100\% dei CFU acquisiti nel periodo pandemico) e quelli del Dipartimento di Storia, Scienze dell'Uomo e della Formazione (DISSUF) (72.7\%); risultano sotto media gli studenti iscritti ai corsi dei dipartimenti di Giurisprudenza (42.6\%), Dipartimento di Scienze Umanistiche e Sociali (DUMAS) $(38.5 \%)$ e Agraria (32.6\%).

\begin{tabular}{|l|c|c|c|}
\hline & CFU 12 mesi $^{8}$ & CFU Covid-19 & $\begin{array}{c}\text { \% CFU Covid / } \\
\text { Anno }\end{array}$ \\
\hline Agraria $=12$ & 132 & 43 & 32.6 \\
\hline DISEA $=2$ & 51 & 51 & 100.0 \\
\hline DISSUF $=23$ & 660 & 480 & 72.7 \\
\hline DUMAS $=4$ & 78 & 30 & 38.5 \\
\hline Giurisprudenza $=10$ & 197 & 84 & 42.6 \\
\hline Totale $=51$ & 1118 & 688 & 61.5 \\
\hline
\end{tabular}

Figura 6. CFU acquisiti su base annua e successivamente al 1.03.2020 dagli studenti del PUP Uniss: dati assoluti per dipartimento universitario e percentuale CFU Covid sul totale.

\subsection{Obiettivi della ricerca}

Partendo dagli elementi contestuali descritti, la ricerca si proponeva di raccogliere e diffondere conoscenze ed evidenze utili alla valutazione dell'andamento della didattica universitaria in ambito penitenziario nel periodo della pandemia sia in termini di efficacia delle misure adottate congiuntamente dall'Università di Sassari e dall'Amministrazione Penitenziaria, sia in termini di soddisfazione percepita da parte degli studenti e degli educatori, in un'ottica di analisi delle criticità riscontrate e di miglioramento delle pratiche didattico-educative e gestionali. Tale obiettivo generale si è declinato nei seguenti due obiettivi specifici:

- rilevare le opinioni ed il punto di vista degli studenti universitari detenuti in merito all'andamento della didattica universitaria durante le restrizioni imposte dalla pandemia da Covid 19, in particolare durante il periodo compreso tra il 5 marzo e il 15 luglio 2020, rispetto a diverse dimensioni riguardanti sia la frequenza e la qualità dei contatti con docenti e personale dell'Università, sia la qualità di erogazione delle attività didattiche (esami e materiali di studio) che l'esperienza di utilizzo delle risorse informatiche e tecnologiche;

- rilevare le opinioni ed il punto di vista degli educatori - denominati Funzionari Giuridico-Pedagogici - in merito al livello di qualità di collaborazione e supporto ricevuto dai docenti e dal personale dell'Università durante le restrizioni imposte dalla pandemia da Covid 19, durante il periodo compreso tra il 5 marzo e il 15 luglio 2020, con particolare attenzione ai principali cambiamenti e criticità incontrate nella gestione dei percorsi didattici universitari degli studenti detenuti, all'utilizzo delle risorse tecnologiche e all'impatto della situazione emergenziale nel lavoro educativo in carcere.

\footnotetext{
${ }^{8}$ Dal 10.08.2019 al 10.08.2020

${ }^{9}$ Dal 01.03.2020 al 10.08.2020
} 


\section{Form@re}

Open Journal per la formazione in rete

\subsection{Campione}

L'indagine ha coinvolto i quattro Istituti Penitenziari che afferiscono al Polo Universitario Penitenziario dell’Università di Sassari, ossia: la Casa Circondariale di Sassari-Bancali; la Casa di reclusione di Alghero; la Casa Circondariale di Nuoro e la Casa di reclusione di Tempio Pausania-Nuchis. All'interno dei suddetti Istituti è stata richiesta la partecipazione degli studenti detenuti iscritti all'Università di Sassari e dei Funzionari GiuridicoPedagogici. In totale, hanno partecipato all'indagine 34 studenti e 5 Funzionari GiuridicoPedagogici. Nella figura 7 si riporta il numero complessivo degli studenti suddivisi per corso di laurea di appartenenza.

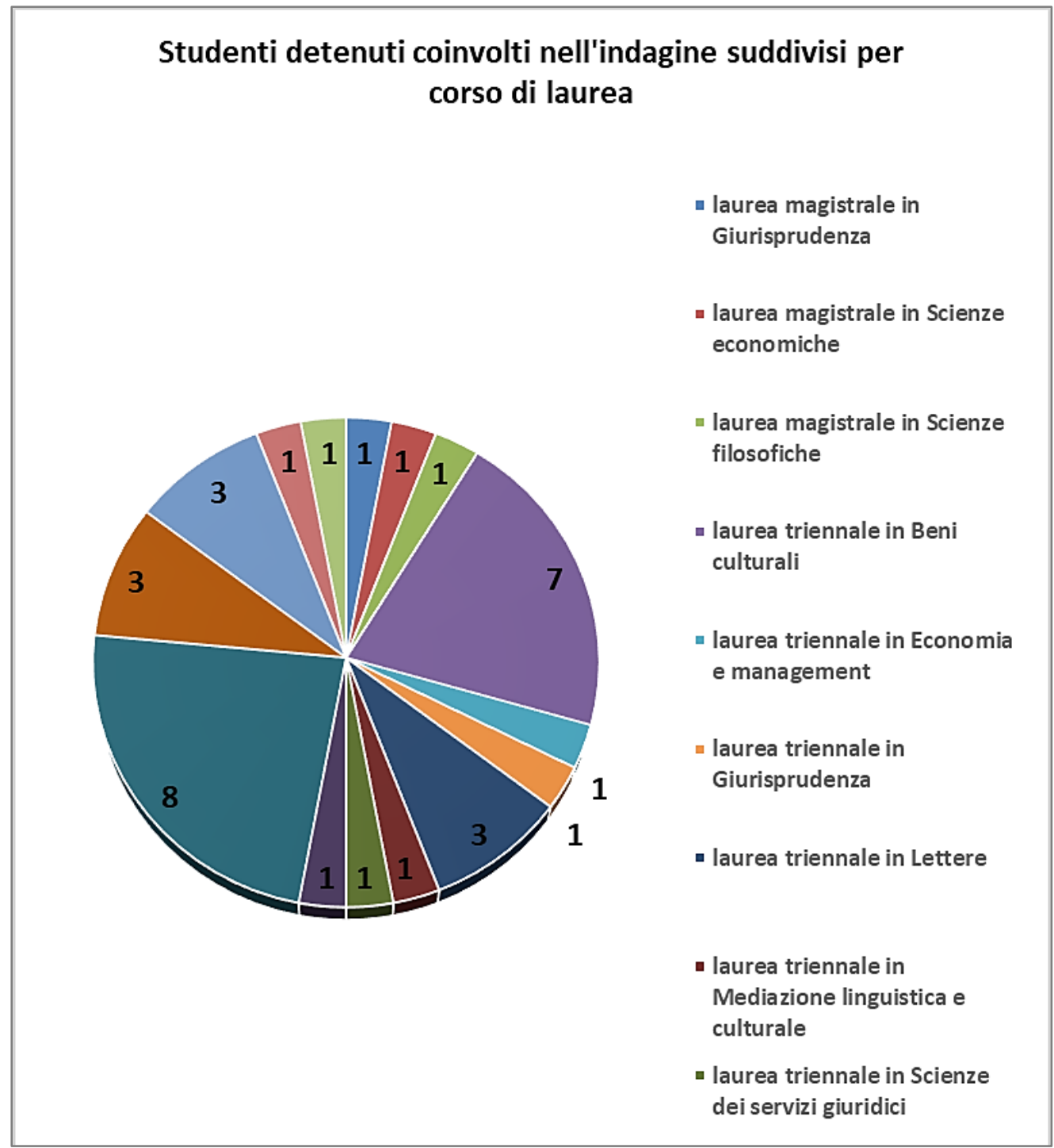

Figura 7. Studenti detenuti coinvolti nell'indagine, suddivisi per corso di laurea di appartenenza.

Come si evince dal grafico, il maggior numero di studenti universitari del campione sono iscritti ai corsi di laurea di Scienze dell'educazione e Scienze dei beni culturali e, a seguire, ai corsi di laurea in Lettere, Scienze dei servizi giuridici e Scienze forestali. 


\subsection{Strumento di ricerca}

Lo strumento di rilevazione dati utilizzato è stato il questionario implementato su supporto cartaceo ed auto-compilato dai soggetti del campione in modo anonimo. Il questionario rivolto agli studenti detenuti si articolava in otto item formulati sia attraverso scale di Likert che domande di scelta e domande aperte. Il questionario rivolto ai Funzionari GiuridicoPedagogici si articolava in sette item formulati sia attraverso scale di Likert che domande aperte. In entrambi i questionari era previsto uno spazio finale dedicato ad eventuali osservazioni e commenti liberi. I questionari sono stati inviati via mail alle Direzioni dei quattro Istituti Penitenziari già menzionati nel mese di luglio 2020 e grazie alla collaborazione dell'Area Educativa-Trattamentale di ciascun Istituto sono stati somministrati e compilati dagli studenti e dagli educatori che hanno scelto di partecipare all'indagine. I questionari compilati sono stati raccolti ed inviati scansionati ai ricercatori sempre da parte dei funzionari delle aree educative degli Istituti Penitenziari entro il mese di ottobre 2020. Pertanto, l'analisi dei dati è stata svolta nel mese di novembre 2020 attraverso il caricamento dei dati quantitativi in matrice dati e la descrizione della loro distribuzione di frequenza e l'analisi e codifica delle variabili testuali dei dati qualitativi.

\subsection{Principali esiti emersi}

Per ciò che concerne le percezioni ed i vissuti degli studenti detenuti, i dati raccolti mostrano come, sebbene nella maggior parte dei casi i contatti con i docenti ed il personale dell'Università siano stati mantenuti durante il periodo marzo-luglio 2020, la comunicazione sia stata difficile e sia avvenuta principalmente attraverso la mediazione degli educatori e l'utilizzo delle piattaforme digitali, come si evince dai grafici sottostanti (Figure 8 e 9).

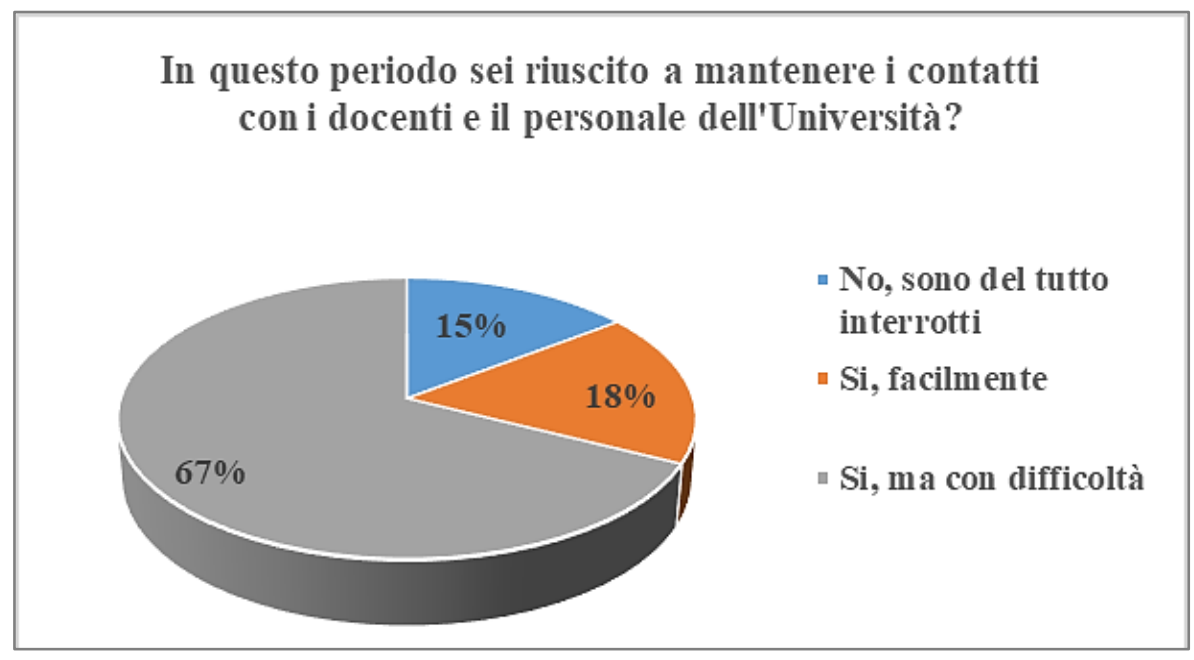

Figura 8. Item 1 del questionario studenti. $\mathrm{N}=34$ questionari compilati. 


\section{Form@re}

Open Journal per la formazione in rete

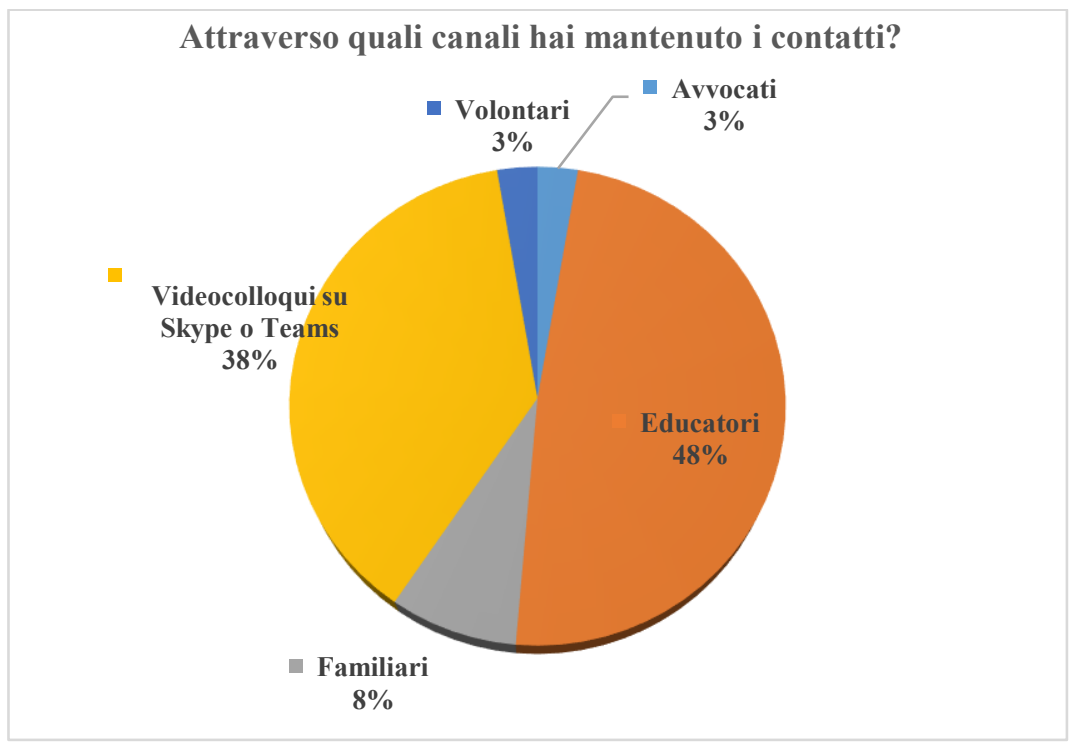

Figura 9. Item 2 del questionario studenti, in cui era consentito indicare più risposte.

Relativamente alla possibilità da parte degli studenti di ricevere i materiali di studio, emerge che, nonostante le restrizioni imposte dalle misure di contrasto e prevenzione della pandemia, la maggior parte degli studenti è riuscita ad avere i materiali necessari sia in forma cartacea che digitale (Figure 10 e 11). Chiaramente le differenze di risposta attengono anche alle diverse situazioni organizzative degli Istituti Penitenziari e alla loro capacità di attivare e mantenere contatti più o meno regolari con docenti e tutor, come spiegato nel paragrafo 4.1 .

\section{In questo periodo sei riuscito a ricevere i materiali di studio?}

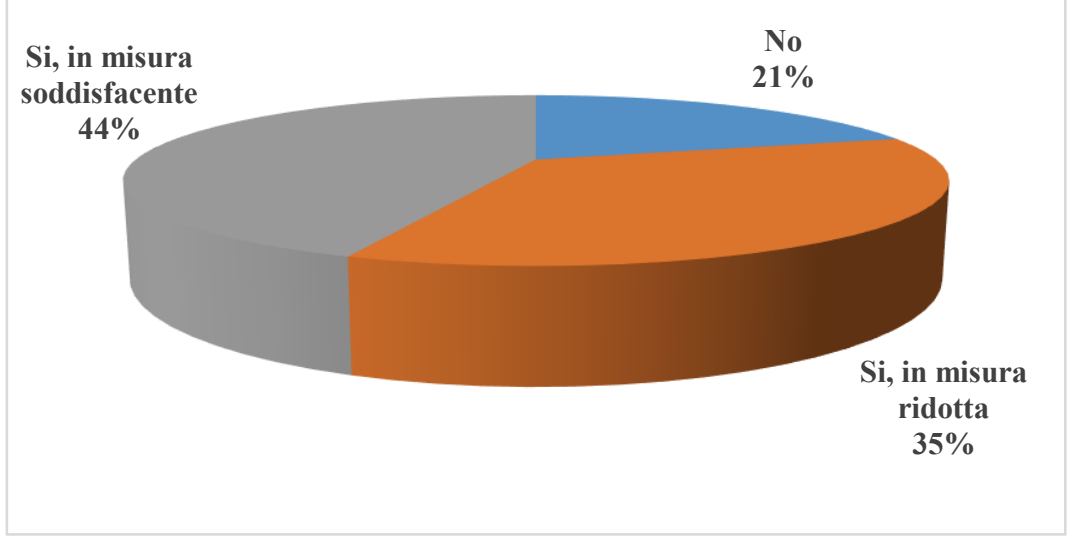

Figura 10. Item 3 del questionario studenti. 


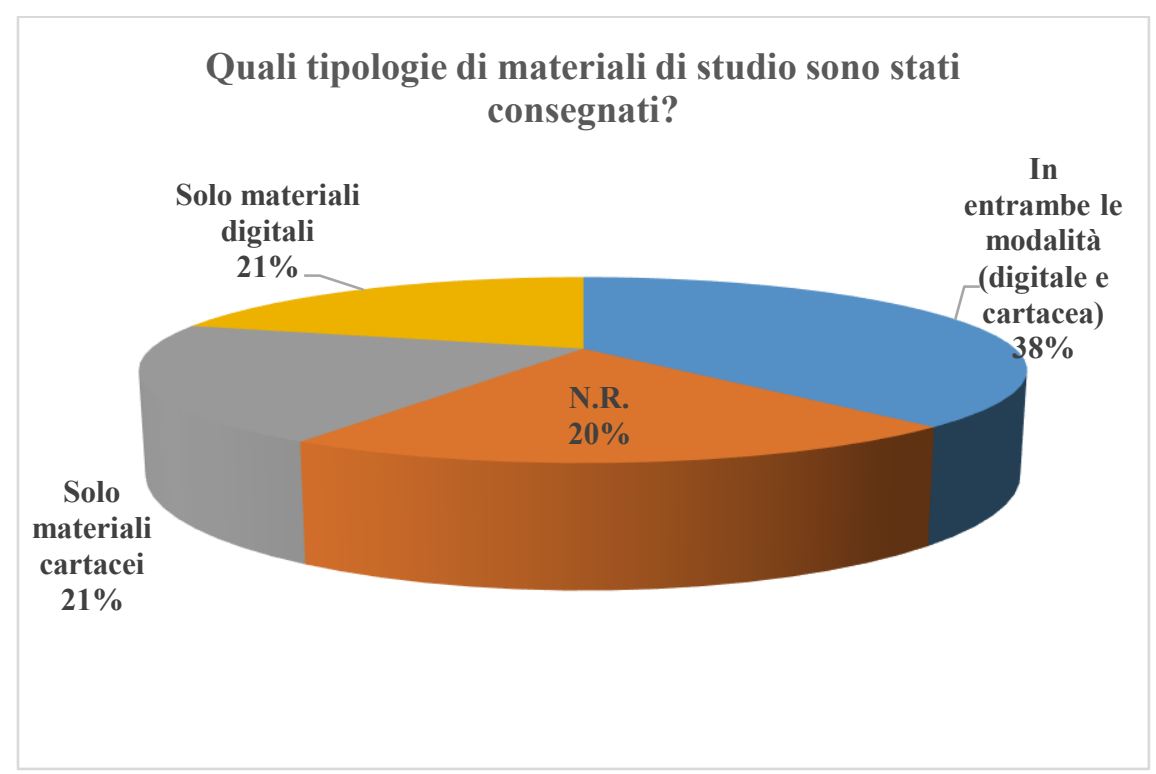

Figura 11. Item 4 del questionario studenti. La sigla N.R. indica "nessuna risposta" e si riferisce a coloro che hanno dichiarato di non aver ricevuto nessun materiale di studio.

Le risposte agli item 5 e 6 del questionario (Figure 12 e 13) mettono in luce come la maggior parte degli studenti del campione abbia avuto la possibilità di sostenere gli esami in misura abbastanza soddisfacente sia attraverso prove scritte che orali a distanza. Questi dati sono in linea con le performance degli studenti, in termini di CFU conseguiti durante il periodo interessato dalla pandemia, dettagliate nel paragrafo 4.1. Chiaramente gli studenti che dichiarano di non aver sostenuto esami non hanno fornito alcuna risposta alle modalità di svolgimento degli stessi.

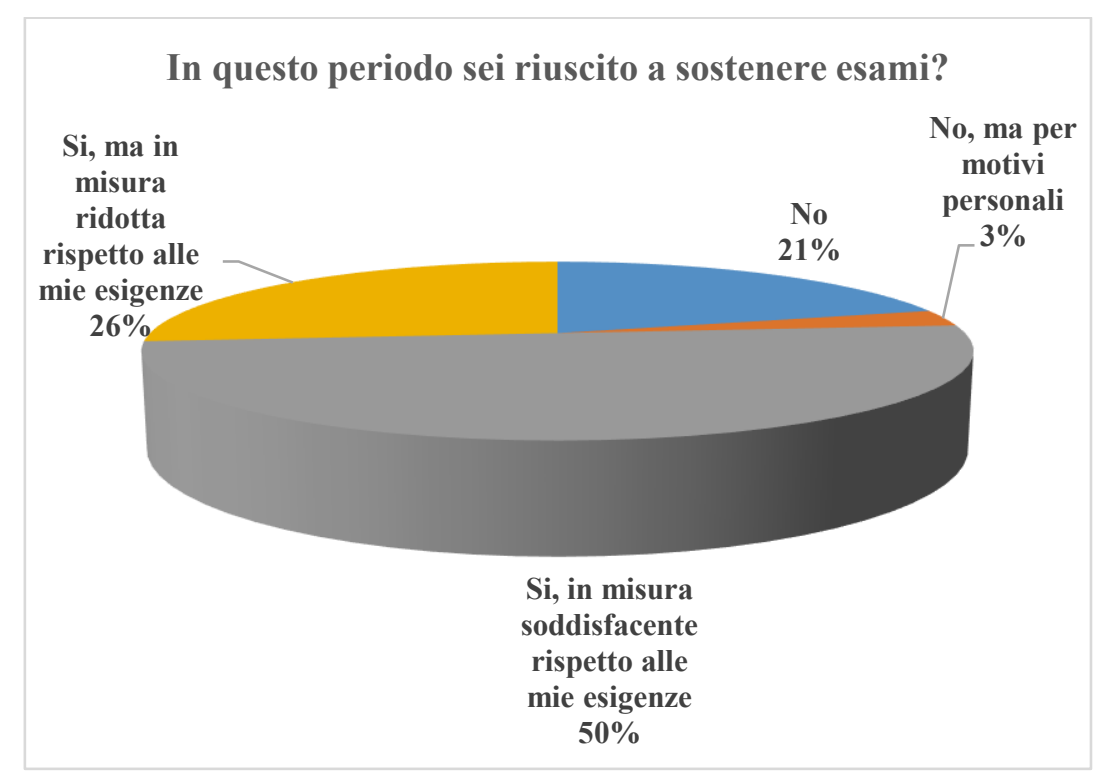

Figura 12. Item 5 del questionario studenti. 


\section{Form@re}

Open Journal per la formazione in rete

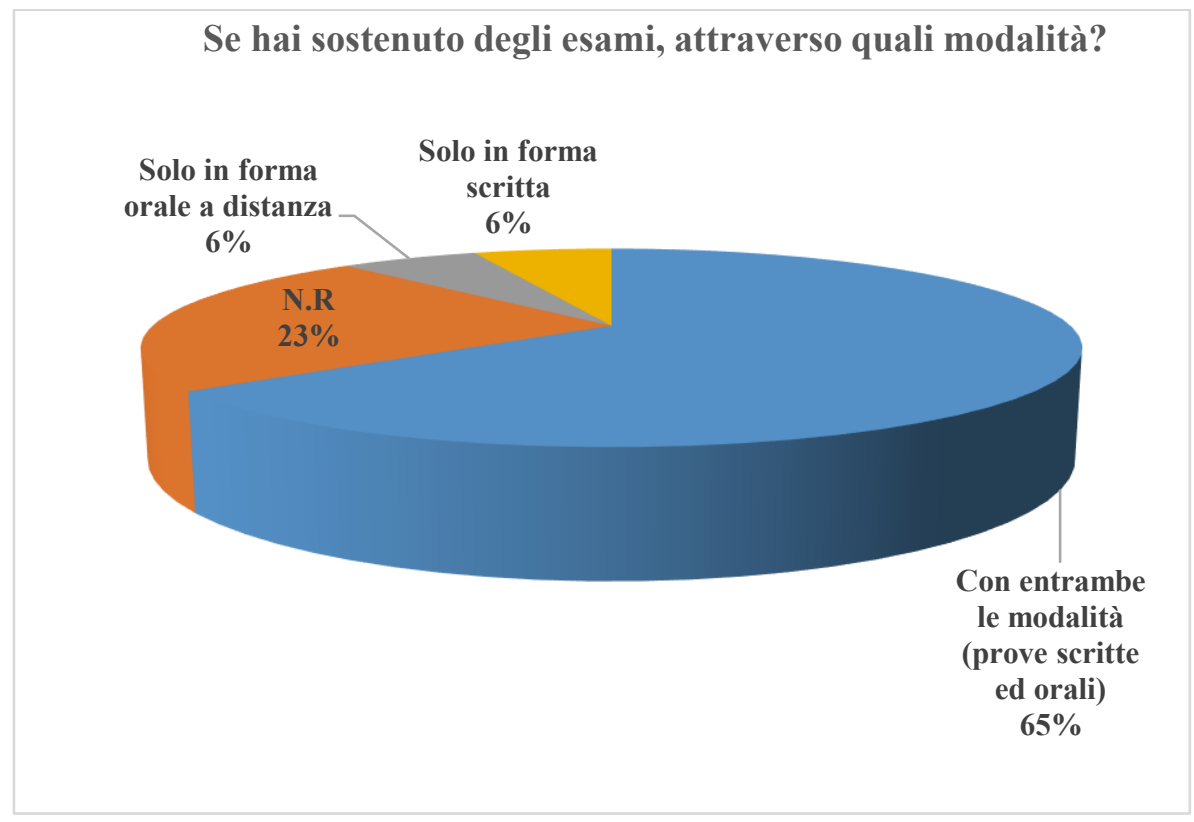

Figura 13. Item 6 del questionario studenti.

In generale, come si evidenzia nel grafico della Figura 14, gli studenti esprimono pareri diversificati rispetto al livello di soddisfazione nei confronti delle modalità organizzative e gestionali implementate dall'Università e dall'Amministrazione Penitenziaria per andare incontro alle loro esigenze didattiche. Anche in questo caso le differenze che emergono sono legate alle situazioni specifiche di ciascun Istituto Penitenziario e ai limiti e difficoltà con cui hanno dovuto fare i conti con risorse e tempi non omogenei tra loro (Figura 15).

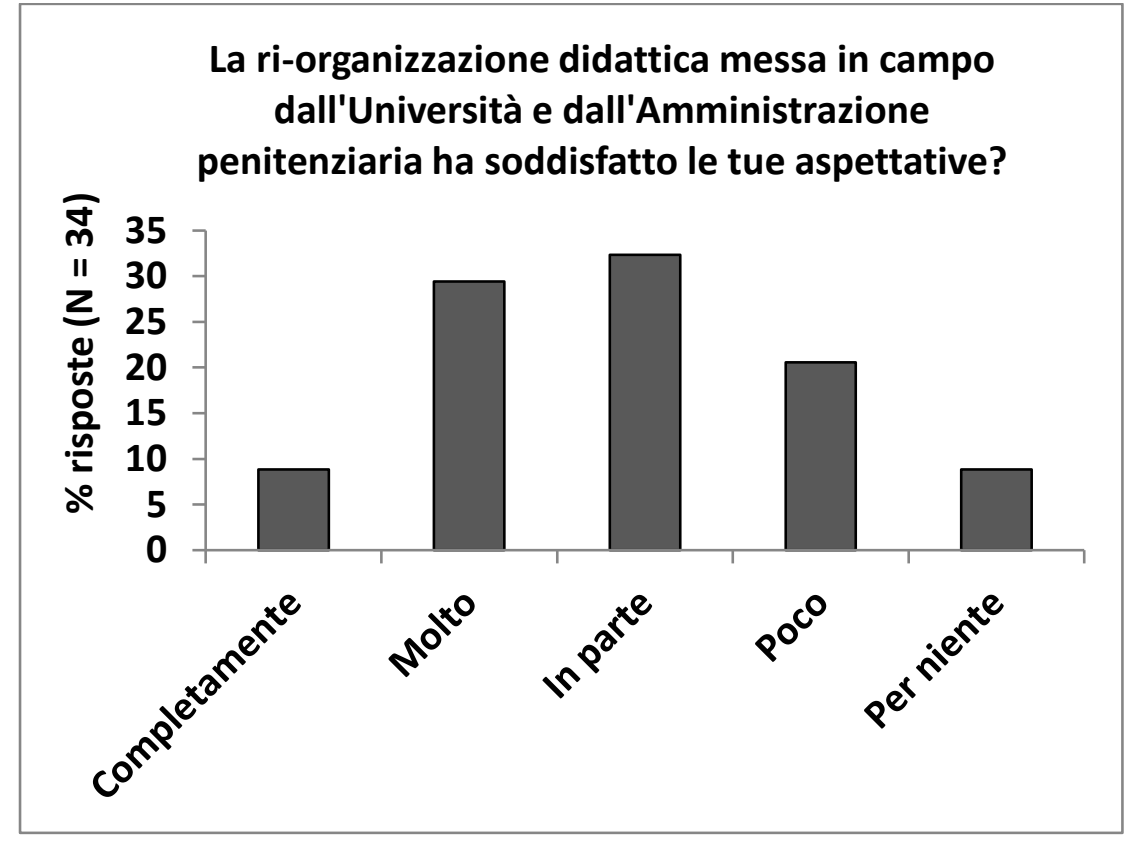

Figura 14. Item 7 del questionario studenti. 


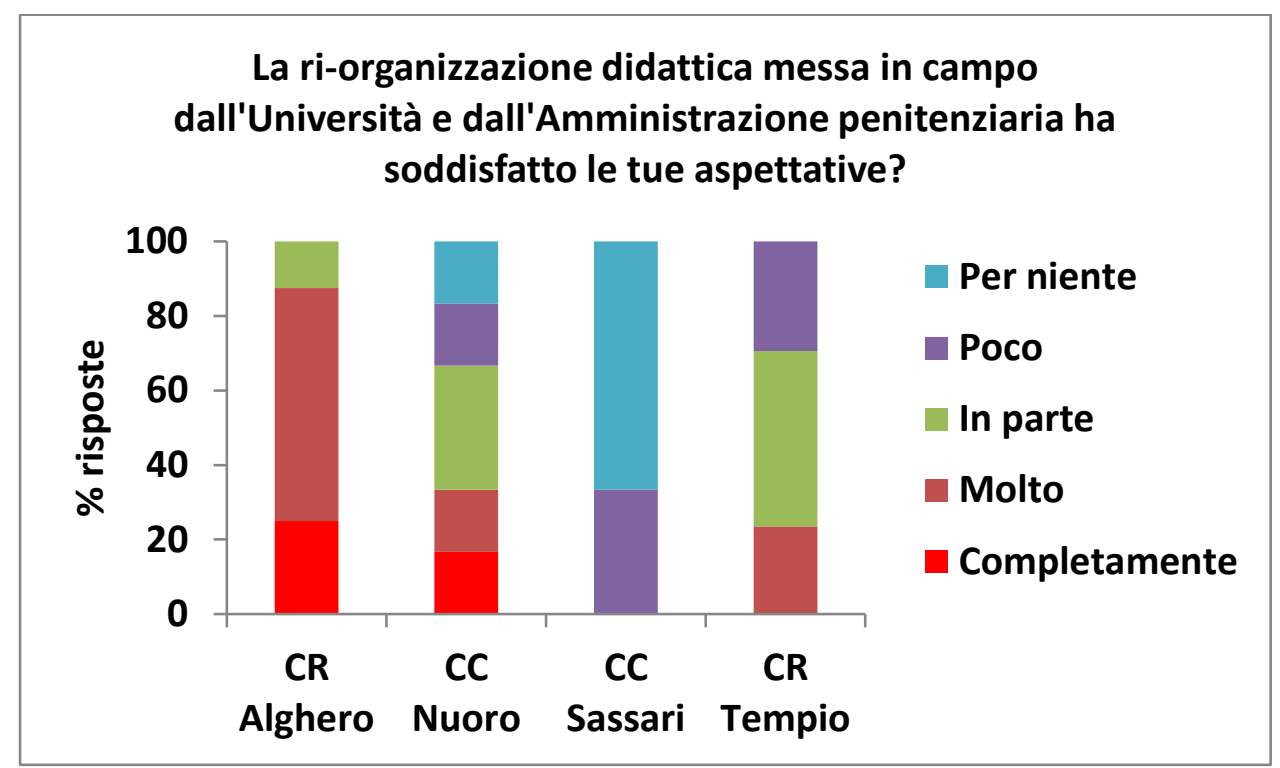

Figura 15. Item 7 del questionario studenti: risposte suddivise per Istituto Penitenziario. CR Alghero: 8 risposte; CC Nuoro: 6 risposte; CC Sassari: 3 risposte; CR Tempio: 17 risposte.

Infine, l'ultima domanda del questionario chiedeva agli studenti di indicare eventuali aspetti positivi legati all'uso delle risorse informatiche nel periodo della pandemia e che potrebbero essere sviluppati anche in futuro. Tutti sono concordi nell'affermare la rilevanza dell'uso delle piattaforme per i collegamenti e le videochiamate con docenti e tutor, da potenziare ed ampliare anche successivamente al periodo dell'emergenza sanitaria, anche al fine di poter avere la possibilità di partecipare, in modalità a distanza, a lezioni e webinar o comunque poter averne la registrazione anche in un momento successivo. In tal senso, gli studenti suggeriscono l'importanza di potenziare il sistema informatico all'interno degli Istituti Penitenziari e la disponibilità di usufruire di dispositivi informatici (pc, tablet, etc.). Molti studenti sottolineano come l'uso delle tecnologie paradossalmente abbia intensificato la relazione ed il confronto con i docenti, anche se in modalità a distanza, rispetto al periodo antecedente alla pandemia, soprattutto per gli studenti detenuti presso gli Istituti Penitenziari geograficamente distanti dalla sede universitaria e più difficilmente raggiungibili dai docenti. In tale prospettiva, auspicano che le risorse informatiche vadano ad integrare sistematicamente, sempre di più, le modalità didattiche tradizionali. Appare interessante soffermarsi anche sui dati emersi dal questionario somministrato ai cinque educatori - Funzionari Giuridico Pedagogici - dei quattro Istituti Penitenziari coinvolti nell'indagine. Relativamente alla qualità della collaborazione con docenti e personale universitario nel periodo marzo-luglio 2020, le risposte variano in base alle singole esperienze degli Istituti Penitenziari. Nelle sedi in cui l'organizzazione interna del carcere ha consentito in tempi brevi di avere a disposizione l'utilizzo delle risorse tecnologiche il livello indicato è buono, mentre nelle sedi in cui questo non è avvenuto, se non con notevole ritardo, si lamenta uno scarso supporto agli studenti e poco confronto tra le due istituzioni. In generale, l'esperienza di utilizzo delle risorse informatiche/tecnologiche da parte degli educatori è stata buona, ma difficile, in quanto l'intero lavoro di 'mediazione' tra il carcere e l'Università, ma anche con i servizi territoriali esterni, i familiari, ecc..., è gravato sull'area educativa-trattamentale, spesso composta da poche unità di personale, se non in alcuni casi uniche, come afferma una delle Funzionarie: "la situazione emergenziale ha di fatto determinato l'essere il riferimento interno per ogni genere di comunicazione con enti 
esterni, non solo con l'Università. Questo, unitamente alla sfortunata coincidenza di essere l'unica risorsa, ha determinato un appesantimento del lavoro in maniera esponenziale. Questo periodo sta determinando la frustrazione che nasce dalla percezione di essere l'unica risorsa di supporto diretto e contemporaneamente non avere la possibilità di esserlo". In generale, per tutto il personale dell'area educativa il lavoro è aumentato nel periodo di riferimento e i principali cambiamenti affrontati riguardano la programmazione e la gestione operativa delle video-chiamate e dei collegamenti con l'esterno. Allo stesso tempo, le professioniste educative mettono in evidenza come la futura predisposizione di un'aula informatica all'interno del carcere dedicata agli studenti universitari detenuti sia una delle potenzialità da sviluppare ed implementare in futuro, con la presenza di figure tecniche specifiche (come tutor d'aula) in grado di supportare e guidare le varie attività e i contatti con l'Università.

\section{Conclusioni}

La ricerca qui presentata offre un dettagliato quadro quali-quantitativo sull'esperienza di didattica universitaria penitenziaria maturata nel periodo pandemico in quattro istituti penitenziari del nord Sardegna, inseriti nel Polo Universitario Penitenziario dell'Università di Sassari. Il dato generale, certamente positivo (in media più del $60 \%$ dei CFU annui sono stati acquisiti dagli studenti detenuti durante i 5 mesi di pandemia), non deve però nascondere le eterogeneità di performances, e quindi di soddisfazione percepita (ed espressa nei questionari), dovute sia a fattori legati all'istituto e al circuito detentivo, sia al percorso universitario (anno e tipologia di corso di studi). In particolare, si nota come la percentuale più alta di CFU acquisiti durante la pandemia sia stata quella degli studenti detenuti in regime ex 41 bis O.P. e nel circuito di Alta Sicurezza, mentre gli studenti detenuti in media sicurezza (cosiddetti detenuti comuni) e quelli in esecuzione penale esterna abbiano avuto performances inferiori alla media. Questo dato del periodo pandemico è, come si è visto, esattamente opposto al trend evidenziato dal monitoraggio effettuato nell'ultimo anno accademico precedente la pandemia (2018-19) e mostra come i detenuti in regime di 41 bis e i detenuti AS sono probabilmente già adattati a situazioni di isolamento/distanziamento rispetto agli altri, oltre che trovarsi all'interno di programmi trattamentali basati su impegni più lunghi e strutturati nel tempo. Alla luce di ciò si può ipotizzare che i processi di resilienza rispetto ai forti disagi generati dalle misure restrittive adottate per il contrasto della pandemia, siano stati maggiori da parte di quei segmenti della popolazione studentesca detenuta (41bis e $\mathrm{AS}$ ) che più frequentemente (ordinariamente nel caso dei 4lbis) sperimentano restrizioni che arrivano fino all'ergastolo ostativo: è facilmente desumibile che questa tipologia di detenuti studenti abbia maggiori abilità e competenze nel gestire situazioni di scarsità di contatti e limitato accesso alle informazioni e ai materiali di studio, e ottimizzi meglio le poche occasioni di contatto (ad esempio: colloqui ed esami orali) che si presentano. Al contrario gli altri due gruppi di studenti in regime di detenzione (quelli in media sicurezza e in esecuzione penale esterna) derivano generalmente da percorsi detentivi meno restrittivi, in cui la possibilità di contatti diretti con docenti e tutor, l'accesso ad informazioni e a materiali di studio sono più facili e frequenti, mentre la dematerializzazione è normalmente un'eccezione piuttosto che la norma (come invece accade soprattutto nei reparti 4lbis e secondariamente nei circuiti AS). La forza dirompente della pandemia tutt'ora in corso, nel contesto penitenziario di riferimento, è stata proprio quella di rivelare punti di forza e di debolezza altrimenti sconosciuti durante $\mathrm{i}$ periodi di gestione ordinaria delle attività didattiche universitarie, $\mathrm{e}$ di capovolgere gli esiti di performance annuale dei diversi segmenti di popolazione 
studentesca detenuta sulla base di abilità e competenze pre-acquisite, correlate soprattutto al regime detentivo e all'istituto penitenziario di appartenenza. Il consolidamento ed il potenziamento della didattica universitaria in carcere richiede quindi l'attivazione di un approccio trasformativo ed innovativo a livello metodologico ed organizzativo che sappia promuovere sinergie ed inter-professionalità tra le varie istituzioni coinvolte. In questa direzione si muove il già citato Protocollo d'intesa recentemente siglato dall'Università di Sassari, il Provveditorato Regionale dell'Amministrazione Penitenziaria, l'Ufficio Interdistrettuale di Esecuzione Penale Esterna di Cagliari e il Centro per la Giustizia Minorile per la Sardegna. Un documento in cui particolare attenzione viene riservata all'implementazione di adeguati spazi da dedicare, all'interno degli Istituti Penitenziari, alle attività di studio e didattica universitaria, anche mediante la dotazione di strumenti $\mathrm{e}$ risorse informatiche e tecnologiche. Le molteplici sfide che la pandemia ha posto all'istruzione assumono una valenza ancora maggiore all'interno del sistema della giustizia; le evidenze emerse dall'indagine sul campo richiamano la necessità di sviluppare una maggiore integrazione di qualità tra tecnologia e processi di insegnamento e apprendimento, che sappia andare oltre la logica emergenziale, rinforzando i passi compiuti fin qui e sostenendoli in modo più articolato, strutturato e sostenibile. In tal senso, possibili sviluppi dell'indagine potrebbero andare nella direzione di esplorare l'impatto e il ruolo della formazione universitaria nel percorso di vita della persona detenuta, con un approfondimento delle traiettorie biografiche degli studenti detenuti nella correlazione con la decisione di intraprendere il percorso accademico e, nello specifico, rispetto alla tipologia di corso di laurea scelto; così come l'opportunità di coinvolgere in modo più attivo i Funzionari Giuridico Pedagogici, partendo anche da problemi e questioni avvertite come significative nella prassi professionale, mediante la costruzione partecipata di progetti di ricerca-azione condivisi.

\section{Riferimenti bibliografici}

Brancucci, M. (2016). La formazione umana in carcere: il ruolo chiave dell'educatore. Formazione, lavoro, persona, $V I(17), 38-46$.

Caldin, R. (2018). Università e carcere: una sfida pedagogica. In V. Friso \& L. Decembrotto (Eds.), Università e carcere. Il diritto allo studio tra vincoli e progettualità (pp. 13-20). Milano: Guerini Scientifica.

Cesaro, A. (2018). La rieducazione tra utopia e possibilità. In V. Friso \& L. Decembrotto (eds.), Università e carcere. Il diritto allo studio tra vincoli e progettualità (pp. $21-$ 36). Milano: Guerini Scientifica.

Comitato Didattico del PUP Uniss (2019). Relazione annuale 2018-19. https://www.uniss.it/sites/default/files/relazione annuale pup 201819 carta intestata.pdf (ver. 15.04.2021).

Comitato Didattico del PUP Uniss (2020). Relazione annuale 2019-20. https://www.uniss.it/sites/default/files/relazione_annuale_pup_2019-20_def.pdf (ver. 15.04.2021).

Decembrotto, L. (2018). Istruzione e formazione in carcere: università, competenze e processi inclusivi. Lifelong Lifewide Learning, 14(32), 108-119.

Decreto Ministeriale 9 agosto 2017, n. 610. Criteri di ripartizione del Fondo di Finanziamento Ordinario (FFO) per l'anno 2017. 


\section{Form@re}

Open Journal per la formazione in rete

D. Lgs. 2 ottobre 2018, n. 123. Riforma dell'ordinamento penitenziario, in attuazione della delega di cui all'articolo 1 , commi 82, 83 e 85, lettere a), d), i), 1), m), o), r), t) e u), della legge 23 giugno 2017, n. 103. (18G00149). Gazzetta Ufficiale Serie Generale n. 250 del 26-10-2018 - Suppl. Ordinario n. 50.

Downes, P. (2014). Access to Education in Europe: A Framework and Agenda for System Change. London: Springer.

Farris, E., \& Guido, V. (2020). Il PUP Uniss. In E. Farris \& P. Sechi (Eds.), Dentro \& Fuori-Atti del workshop formativo e informativo sui percorsi da e per il carcere in Italia (pp. 293-296). Napoli: Jovene.

MIUR. Ministero dell'Istruzione, dell'Università e della Ricerca, Decreto 12 marzo 2015 Linee guida per il passaggio al nuovo ordinamento a sostegno dell'autonomia organizzativa e didattica dei Centri provinciali per l'istruzione degli adulti. https://www.gazzettaufficiale.it/eli/id/2015/06/08/15A04226/sg (ver. 15.04.2021).

Knowles, M. (1993). Quando l'adulto impara. Pedagogia e andragogia. Milano: Franco Angeli.

Pandolfi, L. (2020). Giovani adulti, progettualità e resilienza: ruolo e finalità dell'intervento pedagogico. In E. Farris \& P. Sechi (Eds.), Dentro \& Fuori - Atti del workshop formativo e informativo sui percorsi da e per il carcere in Italia (pp. 73-78). Napoli: Jovene.

Prina, F. (2018). I Poli universitari penitenziari in Italia. L'impegno delle università per il diritto allo studio dei detenuti. In V. Friso \& L. Decembrotto (eds.), I Poli universitari penitenziari in Italia. L'impegno delle università per il diritto allo studio dei detenuti (pp. 87-117). Milano: Guerini Scientifica.

Prina, F. (2020a). L'impegno delle Università nelle istituzioni penitenziarie: diritto dei detenuti agli studi universitari, ricerca e terza missione. Autonomie locali e servizi sociali - Quadrimestrale di studi e ricerche sul welfare, 1(2020), 209-212.

Prina, F. (2020b). La Conferenza Nazionale dei Poli Universitari Penitenziari Italiani. In E. Farris \& P. Sechi (Eds.), Dentro \& Fuori - Atti del workshop formativo e informativo sui percorsi da e per il carcere in Italia (p. 285). Napoli: Jovene. 